\title{
Assisted reproduction techniques in horses - clinical application by different programs around the world
}

\author{
Carolina Herrera \\ Clinic of Reproductive Medicine, Vetsuisse-Faculty, University of Zurich, Switzerland
}

\begin{abstract}
Summary: Assisted Reproductive Techniques (ARTs) in horses has seen a fast growth in the last few yers. Some of the ARTs which are currently being used in the equine around the world are: embryo transfer, intracytoplasmic sperm injection (ICSI), nuclear transfer, oocyte transfer, preimplantation genetic diagnosis (PGD), embryo cryopreservation and sexed semen. The collection of embryos by uterine flushing followed by embryo transfer is a successful technique which has been widely accepted in many horse breeds and is used in various countries around the world. One of the fastest growing techniques is Intracytoplasmic Sperm Injection (ICSI) in combination with Ovum Pick Up (OPU). In 2016, ten equine reproduction programs around the world included OPU-ICSI in their services. In 2017, five more groups were added to this list. Cloning by Somatic Cell Nuclear Transfer (SCNT) has been successfully used by different groups in Argentina, Brazil, Canada, Colombia, Korea, Italy and United States. Nowadays the cloning industry is mainly centered in South America, in Argentina, Brazil and Colombia, where slaughterhouse ovaries are widely available and the maintenance costs for a large recipient herd are much low, compared to countries from North America or Europe. Oocyte transfer (OT) has been applied by three different clinical programs around the world. After the recent increase of the efficiency of the OPU-ICSI technique, OT is less frequently used. Preimplantation Genetic Diagnosis (PGD) has been mainly used in equine embryos to determine the sex of the embryo. In some breeds, one sex is preferred over the other and by means of PGD, the need to produce pregnancies from the undesired sex can be avoided. Additionally, PGD has also been used to detect specific mutations associated with genetic disorders or genes associated to specific phenotypes as, for example, coat color. Until recently, only small $(<300 \mu \mathrm{m})$ equine in vivo derived embryos could be cryopreserved. In 2011 , the successful vitrification of large embryos $(>300 \mu \mathrm{m})$ was described for the first time after collapsing the blastocoele cavity before vitrification. Anyway, this technique is not being used in large-scale commercial programs. Although sexed semen by flow cytometry is widely included in cattle production systems, its use in equine programs presents some disadvantages. In September 2017, the preliminary results produced after selection of equine female sperm cells by means of magnetic nanoparticles were reported. These results are extremely encouraging, since the technique is simple, efficient and does not affect sperm viability, even after freezing and thawing. The aim of the present work is to present an update of the use of ARTs in equine reproduction programs around the world.
\end{abstract}

Keywords: Assisted Reproduction Techniques, Equine, Embryo Transfer, OPU, ICSI, Cloning, Oocyte Transfer, PGD, Embryo Cryopreservation, Sexed Semen

Citation: C. Herrera (2018) Assisted reproduction techniques in horses: clinical application by different programs around the world. Pferdeheilkunde 34, 47-50; DOI 10.21836/PEM20180108

Correspondence: Dr. Carolina Herrera, Clinic of Reproductive Medicine, Vetsuisse-Faculty, University of Zurich, Winterthurerstrasse 260, 8057 Zürich, Switzerland; cherrera@vetclinics.uzh.ch

\section{Introduction}

The application of Assisted Reproductive Techniques (ARTs) to the equine industry has seen a great progress during the last decade. Some of the ARTs which are currently being used in the equine around the world are: embryo transfer, intracytoplasmic sperm injection (ICSI), nuclear transfer, oocyte transfer, preimplantation genetic diagnosis (PGD), embryo cryopreservation and sexed semen. Horse breeders from many countries have demonstrated a recent and increasing interest in applying these techniques to produce foals. Therefore, the aim of this work is to present an update on the ARTs that are being used in equine commercial programs around the world.

\section{Embryo Transfer}

This technique involves the uterine flushing and recovery of an embryo from a donor mare which was inseminated with fresh or frozen semen 8 days before collection. The recovered embryo is then transferred to the uterus of synchronized recipient mare. The collection of embryos by uterine flushing followed by embryo transfer is a successful technique which has been widely accepted in many horse breeds and is used in various countries around the world. According to the last annual report from the International Embryo Technology Society (IETS), during 2015 a total of 21,321 equine embryos were collected by uterine flush. These results include the embryos obtained in Argentina, Brazil, Canada, France, Hungary, Mexico, Poland, South Africa, Sweden, Switzerland and United States (IETS Newsletter, 2016). This number is underestimated, since countries like Chile, Colombia, Germany, Italy, Spain and The Netherlands did not report their numbers, but are well known for their equine embryo transfer activity. Additionally, Argentina only reported 1505 embryos, which probably represents only one tenth of the real embryo production. Therefore, although embryo transfer is a widely accepted technique among veterinarians and breeders, an accurate calculation of the total number of embryos produced around the world is not possible so far.

\section{Ovum Pick Up (OPU) and Intracytoplasmic Sperm Injec- tion (ICSI)}

Intracytoplasmic Sperm Injection (ICSI) in combination with Ovum Pick Up (OPU) is probably the ART that has seen the 
fastest growth in the last five years. In the OPU procedure, the oocytes are collected from a donor mare with a needle connected to an aspiration pump, which is used to puncture the follicles. The needle is guided into the mare transvaginally with a probe, which also holds and ultrasound transducer that allows the visualization of the follicles during the procedure. The follicular fluid containing the oocytes is collected in a bottle or tube and the oocytes are recovered in the laboratory under a stereomicroscope. Once at the laboratory, the in vitro production process begins once the oocytes are placed in in vitro maturation medium for 30 hours, under controlled conditions in an incubator. After this, all mature oocytes are injected with one sperm and placed back in the incubator for in vitro culture for 8 more days. In other mammalian species like cattle or pigs, conventional In Vitro Fertilization (IVF) can be used to produce embryos in the laboratory. Conventional IVF has proven to be unsuccessful to produce equine embryos. Therefore, ICSI is the only technique available for horses. The efficiency of in vitro production of equine embryos by ICSI has improved considerably and the embryo production rates are now compatible with commercial programs (Colleoni et al. 2007, Galli et al. 2007, Foss et al. 2013, Hinrichs et al. 2014). It is nowadays possible to produce between 0.7 to 1 equine embryo in vitro per OPU session. Some of the advantages of this technique are:

- OPU-ICSI can be performed throughout the year, every 14 days on each mare.

- One straw of frozen semen can be used to fertilize more than 100 oocytes by ICSI in different sessions, by thawing only a portion of the straw each time.

- In vitro produced equine embryos are able to survive cryopreservation and thawing successfully

- OPU-ICSI can be performed in young mares, causing a significant reduction in generational intervals

The biggest challenge of this technique is to establish a successful in vitro embryo production laboratory. Although different authors describe in detail the protocols used for in vitro production of equine embryos, there are almost no reports analyzing important details of the laboratory setup, such as quality control and management of the physical and chemical conditions. The management of these conditions is, most of the times, the secret for the success or the reason for failure in embryo production (Herrera et al. 2016). Setting up an in vitro production laboratory is not mandatory to apply OPU-ICSI in an equine reproduction program. Immature oocytes collected from small follicles can be shipped at room temperature to and in vitro production laboratory. The viability of the oocytes is maintained if the temperature remains stable during the transport to the laboratory and the oocytes arrive within 24 hours. In 2016, ten equine reproduction programs around the world included OPUICSI in their services. In 2017, five more groups were added to this list. Until the end of 2017, OPU-ICSI is being performed in Argentina, Belgium, Brazil, Colombia, Italy, Switzerland, The Netherlands and United States. Both OPU and ICSI are used in clinical programs to produce embryos from infertile mares and stallions, young mares, deceased stallions from which very few semen samples are available and semen samples that do not qualify for artificial insemination.

\section{Cloning by somatic cell nuclear transfer (SCNT)}

In order to produce an individual by SCNT, one somatic cell from a donor animal is fused to an enucleated oocyte. Oocytes collected from slaughterhouse ovaries or by OPU can be used for this technique. The enucleated oocyte transferred with a somatic cell is then artificially activated to induce the reprogramming of the new couplet to form a viable embryo. This embryo will contain the same genetic information of the donor from which the somatic cell was collected. The first individual from the Equus caballus species produced by SCNT was born in Italy in 2003 (Galli et al. 2003). Since then, this technique has been successfully used by different groups in Argentina, Brazil, Canada, Colombia, Korea, Italy and United States. In order to produce one viable foal by SCNT, hundreds of oocytes collected from slaughterhouse ovaries and a large recipient herd are needed, since the SCNT procedure is still quite inefficient and pregnancy losses can occur throughout gestation. Nowadays the cloning industry is mainly centered in South America, in Argentina, Brazil and Colombia, where slaughterhouse ovaries are widely available and the maintenance costs for a large recipient herd are much low, compared to countries from North America or Europe.

\section{Oocyte Transfer (OT)}

Oocyte Transfer involves the collection of an oocyte from a preovulatory follicle from a donor mare by OPU or flank aspiration. The recovered oocyte is surgically transferred into the oviduct of an inseminated recipient mare. So far, this technique has been applied by three different clinical programs around the world; two from United States and one from Argentina (Carnevale et al. 2005, Hinrichs et al. 1998, Riera et al. 2016). Before the production of equine embryos by ICSI became efficient, oocyte transfer emerged as an alternative to produce embryos from infertile mares. Oocyte Transfer is generally used for infertile mares that do not produce an embryo or a pregnancy using other techniques like artificial insemination and embryo transfer. After the recent increase of the efficiency of the OPU-ICSI technique, OT is less frequently used. During OT, the oocyte is fertilized in the oviduct and early embryo development occurs in the recipient mare. This constitutes a more natural method to produce a viable embryo, when compared to in vitro embryo production. Anyhow, OT presents two technical disadvantages: the need of a surgical procedure to transfer the oocyte into the oviduct and the need of one whole semen dose for each oocyte.

\section{Preimplantation Genetic Diagnosis (PGD)}

Preimplantation Genetic Diagnosis involves the collection of one or more cells from a preimplantation embryo. The biopsy sample is then analyzed by molecular biology techniques such as Polymerase Chain Reaction (PCR) and the result allows the breeder to decide whether to transfer the embryo or not. PGD has been mainly used in equine embryos to determine the sex of the embryo. In some breeds, one sex is preferred over the other and by means of PGD, the need to produce pregnancies from the undesired sex can be avoided. Additionally, PGD has also been used to detect specific mutations associated with genetic disorders (Choi et al. 2010, 
Choi et al. 2015) or genes associated to specific phenotypes as, for example, coat color (Guignot et al. 2015). PGD has been successfully incorporated to a commercial embryo transfer program, to select the female embryos produced from Polo Argentino horses (Herrera et al. 2014). In cattle, PGD is used in combination with SNPs (Single Nucleotide Polymorphism) chips to predict the milk production or meat tenderness starting from in vitro produced or in vivo derived embryos. Therefore, cattle producers can predict important production characteristics of the individual even before a pregnancy is established. Recently, equine SNPs chips have become available, and will probably be used in the near future to predict the athletic ability of an early embryo or foal.

\section{Cryopreservation of in vivo derived equine embryos}

Until recently, only small $(<300 \mu \mathrm{m})$ equine in vivo derived embryos could be cryopreserved. The large size of the equine embryos and the presence of the capsule which is impermeable to cryoprotectants, where the main reasons why researchers around the world failed to maintain the viability of the embryos after conventional freezing or vitrification. In 2011 , the successful vitrification of large embryos $(>300 \mu \mathrm{m})$ was described for the first time, after collapsing the blastocoele cavity before vitrification (Choi et al. 2011 ). Although embryo cryopreservation allows the transport and maintenance of embryos for large periods of time, enhancing embryo commercialization and storage, this technique is not being used in large-scale commercial programs. A major limitation for the use of embryo cryopreservation of large embryos, is the need of an inverted microscope equipped with micromanipulators and microinjectors and trained personnel to operate this equipment, in order to perform the collapse of the blastocoele cavity.

\section{Sexed Semen}

Cell sorting by flow cytometry detects the difference in the DNA content between male and female sperm and is the most efficient technique used to separate $X$ from $Y$ chromosome bearing sperm cells (Samper et al. 2012). Although sexed semen is widely included in cattle production systems, its use in equine programs presents some disadvantages. For example, sex sorted equine sperm presents low viability after cryopreservation, limiting the use of this technology to centers that own a cell sorter. In 2014, the use of equine sexed semen by flow cytometry was described for the first time in an equine embryo transfer program (Panarace et al. 2014). In September 2017, the preliminary results produced after selection of equine female sperm cells by means of magnetic nanoparticles were reported (Ramirez Castex et al. 2017). The authors demonstrate that exposing equine semen to magnetic nanoparticles does not affect sperm parameters and female sperm were efficiently selected. These results are extremely encouraging, since the technique is simple, efficient and does not affect sperm viability, even after freezing and thawing.

\section{Conclusions}

Assisted reproduction techniques in horses has shown some major progress in the last few years. The efficiency of some of these techniques has been significantly improved recently, such as OPU-ICSI and embryo cryopreservation. Additionally, the acceptance of these techniques by veterinary practitioners and breeders has increased, allowing studies with large number of cases. There is still plenty of room for improvement and the next few years will hopefully bring more success reports.

\section{References}

Carnevale E. M., Coutinho da Silva M. A., Panzani D., Stokes J. E., Squires E. L. (2005) Factors affecting the success of oocyte transfer in a clinical program for subfertile mares. Theriogenology 64, 519-527

Choi Y. H., Gustafson-Seabury A., Velez I. C., Hartman D. L., Bliss S., Riera F. L., Roldan J. E., Chowdhary B., Hinrichs K. (2010) Viability of equine embryos after puncture of the capsule and biopsy for preimplantation genetic diagnosis. Reproduction 140, 893-902

Choi Y. H., Velez I. C., Riera F. L., Roldàn J. E., Hartman D. L., Bliss S. B., Blanchard T. L., Hayden S. S., Hinrichs K. (2011) Successful cryopreservation of expanded equine blastocysts. Theriogenology 76, 143-152

Choi Y. H., Penedo M. C., Daftari P., Velez I. C., Hinrichs K. (2015) Accuracy of preimplantation genetic diagnosis in equine in vivorecovered and in vitro-produced blastocysts. Reprod. Fertil. Dev. 28

Colleoni S., Barbacini S., Necchi D., Duchi R., Lazzari G., Galli C. (2007) Application of ovum pick-up, intracytoplasmic sperm injection and embryo culture in equine practice. Proc. Am. Assoc. Equine Pract. 53, 554-559

Foss R., Ortis H., Hinrichs K. (2013) Effect of potential oocyte transport protocols on blastocyst rates after intracytoplasmic sperm injection in the horse. Equine Vet. J. Suppl. 45, 39-43

Galli C., Colleoni S., Duchi R., Lagutina I., Lazzari G. (2007) Developmental competence of equine oocytes and embryos obtained by in vitro procedures ranging from in vitro maturation and ICSI to embryo culture, cryopreservation and somatic cell nuclear transfer. Anim. Reprod. Sci. 98, 39-55

Galli C., Lagutina I., Crotti G., Colleoni S., Turini P., Ponderato N., Duchi R., Lazzari G. (2003) Pregnancy: a cloned horse born to its dam twin. Nature 424 (6949), 635

Galli C., Lagutina I., Crotti G., Colleoni S., Turini P., Ponderato N., Duchi R., Lazzari G. (2003a) Pregnancy: a cloned horse born to its dam twin. Nature 424, 635

Guignot F., Reigner F., Perreau C., Tartarin P., Babilliot J.M., Bed'hom B., Vidament M., Mermillod P., Duchamp G. (2015) Preimplantation genetic diagnosis in Welsh pony embryos after biopsy and cryopreservation. J. Anim. Sci. 93, 5222-5231

Herrera C., Morikawa M. I., Bello M. B., von Meyeren M., Centeno J. E., Dufourq P., Martinez M. M., Llorente J. (2014) Setting up equine embryo gender determination by preimplantation genetic iagnosis in a commercial embryo transfer program. Theriogenology $81,758-763$

Herrera C., Jeannerat E., Wyck S., Bittner L., Van den Bergh M., Janett F., Burger D., Bollwein H. (2016) Successful in vitro production of mammalian embryos: a strict quality management approach. Proceedings of the International Symposium on Equine Embryo Transfer (ISEET) Meeting, Gent, Belgium, July 2016

Hinrichs K., Matthews G. L., Freeman D. A., Torello E. M. (1998) Oocyte transfer in mares. J. Am. Vet. Med. Assoc. 212, 982-986

Hinrichs K., Choi Y. H., Love C. C., Spacek S. (2014) Use of in vitro maturation of oocytes, intracytoplasmic sperm injection and in vitro culture to the blastocyst stage in a commercial equine assisted reproduction program. J. Equine Vet. Sci. 34, 176

Riera F. L., Roldán J. E., Gomez J., Hinrichs K. (2016) Factors affecting the efficiency of foal production in a commercial oocyte transfer program. Theriogenology 85, 1053-1062

Samper J. C., Morris L., Peña F. J., Plough T. A. (2012) Commercial breeding with sexed stallion semen: reality or fiction? J. Equine Vet. Sci. 32, 471-474 
Statistics of Embryo Collection and Transfer in Domestic Farm Animals (2015) International Embryo Technology Society (IETS). Newsletter, December 2016

Panarace M., Pellegrini R. O., Basualdo M. O., Belé M:, Ursino D. A., Cisterna R., Desimone G., Rodríguez E., Medina M. J. (2014) First field results on the use of stallion sex-sorted semen in a large-scale embryo transfer program. Theriogenology 81, 520-525
Ramirez Castex H., Domínguez E., Flores Bragulat A., Flores S., Pérez Barrios P., Ugaz C., Clemente H., Giojalas L., Losinno L. (2017) Resultados preliminares del sexado de semen equino con nano partículas magnéticas. Memorias de las II Jornadas Internacionales de Biotecnologías Reproductivas en Equinos. September 2017, Pilar, Buenos Aires, Argentina 